\title{
Efecto de la combinación Adara-M2624 y otros siete portainjertos sobre las características del dosel de dos variedades de cerezo
}

Tersoglio, E. y N. Setien

\begin{abstract}
RESUMEN
Se estudió la influencia de los portainjertos SL64, MxM60, MxM14, CAB6P, Weiroot 13, GiSeLaß 5 y GiSeLa ${ }^{\circledR} 6$ y Adara-Mariana 2624, sobre el comportamiento vegetativo de las variedades de cerezo Bing y Celeste, durante 9 años después de la injertación, en las condiciones agroclimáticas locales. El diseño experimental fue por bloques completos al azar, con seis repeticiones de cuatro plantas por unidad experimental. Las variables fueron: área de sección trasversal de la variedad (AST), relación AST variedad-filtro, altura, volumen de dosel, índice de vigor y densidad de sierpes. El tamaño del dosel fue influido principalmente por el portainjerto $y$, en menor medida, por la variedad e interacción. En las condiciones del ensayo, SL64 fue el más vigoroso, seguido por MxM60 como vigoroso. Los MxM14, CAB6P y Adara-M2624 fueron semivigorosos; W13, semienanizante; G6, enanizante y G5, muy enanizante. GiSeLa ${ }^{\circledR}$, GiSeLa ${ }^{\circledR} 6$ y Weirrot 13 mantuvieron el VDct por debajo del máximo disponible, mientras que el de MxM14 y Adara-Mariana 2624 fue controlado sólo con poda. La variedad Bing fue más vigorosa que Celeste. Las combinaciones de portainjertos muy enanizantes con variedades de vigor medio resultaron en árboles demasiado débiles, y viceversa, plantas con excesivo vigor. Los W13, CAB6P y Adara-M2624 formaron sierpes.
\end{abstract}

Palabras clave: cerezo, patrón, área de sección transversal, índice de vigor, sierpes.

Tersoglio, E. and N. Setien, 2016. Effect of Adara-M2624 combination and other seven rootstocks on canopy characteristics of two sweet cherry varieties. Agriscientia 33 (2): 113-125

\section{SUMMARY}

The influence of SL64, MxM60, MxM14, CAB6P, Weirrot 13, GiSeLa® 5, GiSeLa $₫ 6$ and interstocks Adara on Mariana 2624 rootstocks onto vegetative 
growth of Bing and Celeste sweet cherry cultivars was studied under local agro-climatic conditions, during 9 years after grafting. The experimental design consisted of randomized complete block with six replications, with four trees per experimental unit. The variables were trunk cross sectional area of cultivar (TCSA), TCSA ratio (cultivar-interstocks), height, canopy volume, vigor index and root sucker density. The canopy size was influenced mainly by rootstocks and, to a lesser extent, by variety and interaction. Under test conditions, SL64 was the most vigorous, followed by MxM60 as vigorous. MxM14, CAB6P and Adara-M2624 were semi-vigorous, Weiroot 13 was semi-dwarfing, GiSeLa 6 was dwarfing and GiSeLa 5 was very dwarfing. Both GiSeLa and Weirrot 13 kept VDct below the maximum available, while that of MxM14 and AdaraMariana 2624 was controlled just by pruning. Bing cultivar was more vigorous than Celeste. The combination of a very dwarfing rootstocks with medium vigor varieties resulted in very weak trees, and conversely, very vigorous plants. Weiroot13, CAB6P and Adara-M2624 produced root suckers.

Key words: sweet cherry, rootstock, TCSA, vigor index, root suckers.

Tersoglio, E. y N. Setien: Instituto Nacional de Tecnología Agropecuaria (INTA). San Martín 3853, Luján de Cuyo, Mendoza, Argentina. CC3 CP 5507. Correspondencia a: tersoglio.eduardo@inta.gov.ar

\section{INTRODUCCIÓN}

Los cerezos (Prunus avium L.) son árboles grandes, poco inductivos y precoces, lo cual incrementa los costos de producción. En la actualidad existen portainjertos con diferentes cualidades que permiten la adaptación a diversas condiciones edafoclimáticas (Sansavini \& Lugli, 2014). Los resultados de los ensayos entre países e incluso regiones, muestran diferencias (Stehr, 2001; De Salvador et al., 2005; Hilsendegen, 2005; Sitarek et al., 2005). Por ello, previa difusión, es necesario estudiar su desempeño en las condiciones agroclimáticas locales, ya que cada región induce, para un mismo portainjerto, comportamientos muy diferentes (De Salvador et al., 2005; Hilsendegen, 2005; Usenik et al., 2006; Kappel \& Lang, 2008; Long \& Kaiser, 2010; Sansavini \& Lugli, 2014). La información obtenida de estos ensayos permite adaptar el cultivo a las limitaciones de suelo y clima de cada región, ampliando el área de cultivo y modificando las características vegetativas (Guajardo, 2004; Hilsendegen, 2005). Por lo tanto, una apropiada selección es fundamental para optimizar la productividad de los huertos (Usenik et al., 2006; Gonçalvez et al., 2007).

Los portainjertos restrictivos son poco usados en la región (Ojer et al., 2015). Por lo tanto, la identificación del comportamiento vegetativo permitirá difundir los más adecuados según las habilidades y capacidades de los agricultores (Long \& Kaiser, 2010).
Algunos portainjertos producen brotes indeseables, denominados sierpes, que provienen de yemas adventicias ubicadas en las raíces cercanas a la superficie del suelo. Estos son capaces de formar nuevos individuos utilizando las reservas del árbol y, por lo tanto, deben eliminarse tempranamente. Sin embargo, el control químico no permite el uso de herbicidas sistémicos, y el control mecánico o manual produce heridas que permiten la entrada de hongos de madera (Sansavini \& Lugli, 2014)

El objetivo del trabajo fue establecer la influencia de ocho portainjertos sobre las características vegetativas de dos variedades de cerezo, en las condiciones agroclimáticas de Mendoza, Argentina.

\section{MATERIALES Y MÉTODOS}

El ensayo se desarrolló entre 2004 y 2013, en el departamento Luján de Cuyo, Mendoza, Argen-

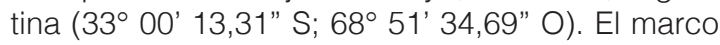
de plantación fue $3 \mathrm{~m}$ entre plantas y $5 \mathrm{~m}$ entre hileras, bajo cubierta antigranizo de $5 \mathrm{~m}$ de altura. Se estudió el comportamiento del dosel de dos variedades sobre ocho portainjertos. El diseño fue por bloques completos al azar, con seis bloquesrepeticiones de cuatro plantas por unidad experimental (Stehr, 2001). Fueron evaluadas sólo plantas sanas, sin síntomas de decaimiento. Los datos analizados fueron los promedios de cada parcela.

El suelo de textura franco arcilloso presentó capas lenticulares horizontales compactadas que 
se intensificaron hacia el bloque V. Las características edáficas de cada bloque fueron: bloque I con CEes 2,88 dS m², VS $109 \mathrm{ml} \% \mathrm{~g}$ y una proporción limo-arcilla ( $L+A)$ 50,5\%; bloque II con CEes 1,71 dS $\mathrm{m}^{-2}$, VS $112,1 \mathrm{ml} \% \mathrm{~g}$ y $\mathrm{L}+\mathrm{A}$ del $55 \%$; bloques III, IV y $\vee$ muestran una CEes de 1,50 a 1,65 $\mathrm{dS} \mathrm{m}^{-2}$ VS entre 114 y $115 \mathrm{ml} \% g$ y $L+A$ entre $57 \%$ y $59 \%$; bloque $\mathrm{VI}$ con una CEes 1,50 dS $\mathrm{m}^{-2}$, un VS 111 $\mathrm{ml} \% \mathrm{~g}$ y un contenido $\mathrm{L}+\mathrm{A}$ del $51 \%$.

El riego, de cuatro líneas de goteo por hilera, aplicó una lámina horaria de 1,9 mm con una reposición fija de 7,6 mm cada vez que el suelo alcanzó un potencial matriz de $30-40 \mathrm{kPa}$.

El contenido de $\mathrm{N}$ total del suelo (método Kjeldahl) fue ligeramente bajo (562 a $627 \mathrm{mg} \mathrm{kg}^{-1}$ ), mientras que el $\mathrm{P}$ disponible (extracción carbónica 1:10) fue muy bajo, menor de $1 \mathrm{mg} \cdot \mathrm{kg}^{-1}$. Los portainjertos fueron plantados en 2003 e injertados en 2004. A partir del 2005, se fertilizaron anualmente con dosis crecientes de 10, 20 y $30 \mathrm{~kg} \mathrm{~N}$.ha ${ }^{-1}$. A partir del 2008, se usó fertirrigación con dosis de reposición que consideraron rendimiento y contenido edáfico. Las dosis de $\mathrm{N}$ oscilaron entre $33 \mathrm{y}$ $70 \mathrm{~kg} \mathrm{~N} \mathrm{ha}^{-1}$, mientras que las de P, entre 22 y $30 \mathrm{~kg}$ $P$ ha-1 en aplicación otoñal (González, 1992; Silva \& Rodríguez, 1995). Además, se realizaron correcciones de Mg, Zn y B según análisis foliar, mediante aspersión primaveral.

El sistema de conducción fue ejes múltiples con tres ramas principales y 18 a 21 laterales. La altura de las plantas fue controlada mediante poda cada vez que superó los 4,5 m. Los rebajes se realizaron sobre ramas insertas aproximadamente a $4 \mathrm{~m}$. No se realizó poda en verde.

En cerezo, la presencia de virus como PDV y PNRSV induce reducciones del crecimiento (Charlot et al, 2005; Pedersen et al, 2005; Long \& Kaiser, 2010), por lo tanto, los materiales utilizados fueron libres de virus.

Las variedades ensayadas fueron: Bing clon OB260 de vigor medio-alto, precocidad media, autoincompatible y Celeste de vigor medio-bajo, precoz y autocompatible.

Los portainjertos ensayados fueron:

Selección SL64 de Prunus mahaleb (SL 64) origen Grande Ferrade, Francia de fácil instalación, buen anclaje y buena compatibilidad. Considerado de alto vigor, alta mortandad (De Salvador et al., 2005; Moreno et al., 1996) y falta de adaptación a suelos pesados (Jiménez et al., 2007; Pina et al., 2011; Lopez-Ortega et al., 2016). Presenta una alta tolerancia a la clorosis y sequía, pero baja a la hipoxia (Long \& Kaiser, 2010).

MxM14 y MxM60 son híbridos inter-específicos
(P. mahaleb $\times$ P. avium), cuyo origen es EEUU. El MxM60 se adapta a diferentes tipos de suelo y clima; es muy vigoroso (Long \& Kaiser, 2010) con sensibilidad intermedia a la hipoxia (Pimentela et al., 2014).

El vigor del MxM14 varía con la región. En Alemania y en Polonia fue considerado enanizante (Stehr, 2001; Grzyb y Sitarek, 2005), en Italia, Portugal, España y EEUU, de vigor intermedio (Moreno et al, 2001; Battistini, 2005; De Salvador et al., 2005; Santos et al., 2006; Long y Kaiser, 2010), mientras que en Eslovenia mostró un comportamiento muy vigoroso (Usenik et al., 2006). Resiste el replante y la clorosis férrica inducida; tiene buen anclaje, es precoz, productivo y medianamente sensible a la sequía y a la hipoxia (Balmer, 2008).

En cuanto a CAB6P y Weiroot 13 (W13), ambas selecciones de Prunus cerasus producen sierpes, adaptados a suelos pesados y calcáreos (Moreno et al., 2001). El CAB6P, de origen italiano, tiene moderada tolerancia a clorosis e hipoxia (Pimentela et al., 2014). El vigor varía con la región en estudio: considerado semivigoroso en EEUU por Webster \& Schmidt (1996) y en Italia por Battistini (2005), De Salvador et al. (2005) y Sansavini \& Lugli (2014), mientras que en España Moreno et al. (2001) y Jimenez et al. (2007) lo consideraron vigoroso. El W13, originario de Alemania, es precoz, productivo, de anclaje intermedio y buena compatibilidad (Webster, 1996; De Salvador et al., 2005), es considerado de vigor intermedio (Stehr, 2001; Kappel et al., 2005; Usenik et al., 2006) y vigoroso (Webster y Schmidt, 1996; Lichiev y Papachatzis, 2009).

GiSeLa® 5 (G5) y GiSeLa® 6 (G6), de origen alemán, son híbridos interespecíficos entre Prunus cerasus x Prunus canescens. El G5 es de bajo vigor (Franken-Bembenek, 2005; Kappel \& Lang, 2008; Long \& Kaiser, 2010; Sansavini \& Lugli, 2014; Lopez-Ortega et al. 2016), aunque Wertheim et al. (1997) y Stehr (2001) lo clasificaron semivigoroso. Se considera que, según las zonas, es de difícil instalación (Neilsen et al., 2010) y alta mortalidad (Kappel \& Lang, 2008; Lopez-Ortega et al., 2016), sensible a clorosis, anclaje intermedio, requiere buen drenaje y no serpea. El G6 es consistentemente de mayor vigor que G5 (Whiting et al., 2005; Long \& Kaiser, 2010), pero de menor vigor que MxM14 y CAB6P (Gratacós et al., 2007). En Estados Unidos mostró comportamientos diferentes: en el noroeste alcanzó el 90\% de un árbol estándar, mientras que en el este sólo el 60\% (Long \& Kaiser, 2010). La mayoría de los autores lo consideran semienanizante (Whiting et al., 2005; Balmer, 2008; Gyeviki et al., 2012). El portainjerto tiene buen anclaje, aunque tiende a inclinarse (Balmer, 2008; Long \& Kaiser, 2010). La planta es fácil de instalar 
y no produce sierpes (Long \& Kaiser, 2010).

Mariana 2624 (Prunus cerasifera x Prunus munsoniana) (M2624) como portainjerto posee un excelente comportamiento en la región, adaptándose a condiciones de hipoxia (Pimentela et al., 2014). Debido a la incompatibilidad del M2624 injertado con cerezo, se utiliza un injerto filtro, el Adara. Este es una selección de Prunus cerasifera var. Mirobalan que es intercompatible con el portainjerto M2624 y con el cerezo (Moreno et al., 1995; Moreno, 2004; Lopez-Ortega et al., 2016). En pruebas previas, Adara autoenraizado mostró cierta mortandad tanto en suelo virgen como en replante, mientras que la combinación M2624-Adara mostró la máxima sobrevivencia (López-Ortega et al., 2016). En suelos pesados, con baja porosidad, se recomienda el uso de portainjertos de ciruelos con filtros intercompatibles con cerezos como M2624Adara (Long \& Kaiser, 2010, López-Ortega et al., 2016). Adara, como portainjerto, induce alto vigor (Cantín et al., 2010), aunque podría reducir el tamaño del dosel al estar utilizado como filtro (Magyar \& Hrotko, 2008; Weibel, 2008).

\section{Indicadores de vigor}

Área de sección transversal del tronco (AST): se calculó a través de la circunferencia del tronco C $10 \mathrm{~cm}$ encima del injerto con la fórmula $A S T=\pi$. $(C / 2 \pi)^{2}$. Los datos analizados correspondieron al período entre el tercer y noveno año después de la injertación (ADI) (2007-2013) (De Salvador et al., 2005; Gonçalves et al., 2007; Jiménez et al., 2007).

Relación AST variedad-filtro (RAST): es el cociente entre el AST de la variedad y el del filtro (Rozpara et al., 1998; Olmstead et al., 2006; Gonçalves et al., 2007; Olmstead et al., 2010) y fue calculado sólo en la combinación P. avium-Adara y medida entre el quinto y el noveno ADI (2009-2013).

Altura $(H)$ : se midió entre el quinto y el noveno ADI (2009-2013). La altura máxima se estableció en 4,50 m, como indicador de riesgo de accidentes laborales con influencia en costos de poda y cosecha (Santos et al., 2006).

Relación área de proyección del dosel y altura (APD-H): el cálculo asume una base circular, utilizando la fórmula $A P D=\pi .((L+E) / 4)^{2}(L$, largo y $E$, espesor) y $\mathrm{H}$. Se midió entre el quinto y el noveno ADI (2009-2013). Se expresa en $\mathrm{m}^{2} \mathrm{~m}^{-1} \mathrm{e}$ indica el área que dispone cada unidad de $\mathrm{H}$ de dosel e interpreta la arquitectura del dosel. Una relación alta sugiere un dosel globoso y viceversa, uno alargado (Santos et al., 2006).

Volumen del dosel $\left(V_{\mathrm{ct}}\right)$ : el cálculo asume la forma de cono truncado. Se utilizó la fórmula $V D_{c t}=$ $\pi \cdot\left(R^{2}+r^{2}+R r\right) \cdot h \cdot 1 / 3$ siendo $R=(E+L) / 2$, mientras que el radio menor ( $r$ ) asumió un valor de 0,40m (Usenik et al., 2006). El VD máximo posible fue 20,78 $\mathrm{m}^{3}$; por ello, los resultados pueden expresarse como una proporción de ese valor máximo (Santos et al., 2006). Se midió entre el quinto y el noveno ADI (2009-2013).

Índice de vigor (IV): es un valor empírico, calculado como el cociente entre la adición del diámetro de tronco $(\mathrm{cm})$ con el volumen del dosel $\left(\mathrm{m}^{3}\right)$ y el promedio anual correspondiente al SL64 (Stehr, 2001), entre el quinto y el noveno ADI.

Densidad de sierpes (DS): es el número de sierpes por unidad de superficie y se expresó como $\mathrm{S} \mathrm{m}^{2}$, medido entre el quinto y el octavo ADI (De Salvador et al, 2005).

Los datos fueron evaluados mediante análisis de la varianza con el programa estadístico InfoStat (Di Rienzo et al., 2015). La prueba de comparación múltiple de medias utilizada fue Scott \& Knott y el nivel de significancia se estableció en $P \leq 0,05$. El coeficiente de correlación utilizado fue el de Pearson. Se establecieron los parámetros del modelo de relación entre el AST y el VD ct a través de los programas informáticos Table Curve 2D (versión prueba) e InfoStat (Di Rienzo et al., 2015). Se estudió el efecto "portainjerto" sobre la relación ASTVDct mediante el método de regresiones anidadas. Éste establece si el origen de las eventuales diferencias AST-VDct de los portainjertos se desarrolla sólo al inicio del crecimiento o se presenta como un proceso progresivo donde la relación AST-VDct se va diferenciando entre los portainjertos (Mendenhall et al., 1995).

\section{RESULTADOSY DISCUSIÓN}

\section{Área de sección transversal del tronco}

El ensayo presentó un AST medio de 117,9 $\mathrm{cm}^{2}$ con una $\mathrm{DE} \pm 109 \mathrm{~cm}^{2}$. El modelo $\left(\mathrm{R}^{2}=0,91\right.$; $P \leq 0,0001)$ mostró que el AST estuvo influenciado por $A D I(P \leq 0,0001)$, portainjerto $(P \leq 0,0001)$, variedad $(P=0,0008)$ e interacciones portainjerto-variedad $(P \leq 0,0001)$, bloque-portainjerto $(P \leq 0,0001)$ y ADI-portainjerto $(P \leq 0,0001)$. Los efectos con mayor poder explicativo fueron ADI (39\%) y portainjerto (33\%).

El AST se incrementó anualmente entre 20 y 36 $\mathrm{cm}^{2}$. Sólo en el noveno ADI se observó un aumento mayor $\left(76,8 \mathrm{~cm}^{2}\right)$ como consecuencia de la ausencia de fruta, que provocó mayor crecimiento vegetativo (Tabla 1). 
Tabla 1. Efecto del ADI, portainjerto, variedad e interacción portainjerto-variedad sobre el AST, RAST, H, APD-H, IV, VD ct y DS de las variedades de cerezo Bing y Celeste.

\begin{tabular}{|c|c|c|c|c|c|c|c|c|}
\hline \multicolumn{2}{|c|}{ Efectos } & ${ }^{2} \mathrm{AST}\left(\mathrm{cm}^{2}\right)$ & ${ }^{3} \mathrm{RAST}$ & $\mathrm{H}(\mathrm{m})$ & ${ }^{4} \mathrm{APD}-\mathrm{H}\left(\mathrm{m}^{2} \mathrm{~m}\right)$ & ${ }^{5} \mathrm{IV}$ & $\begin{array}{l}{ }^{6} D_{\mathrm{ct}} \\
\left(\mathrm{m}^{3}\right)\end{array}$ & ${ }^{7} \mathrm{DS} \mathrm{Sm}{ }^{-2}$ \\
\hline \multirow{7}{*}{${ }^{1} \mathrm{ADI}$} & 3ro & 36,58 a & & & & & & \\
\hline & 4 to & $56,71 \mathrm{~b}$ & & & & & & \\
\hline & 5 to & $76,83 \mathrm{c}$ & $2,53 \mathrm{a}$ & $3,53 \mathrm{a}$ & $1,93 \mathrm{a}$ & $0,71 \mathrm{a}$ & $12,22 \mathrm{a}$ & $0,07 \mathrm{a}$ \\
\hline & 6 to & $101,12 \mathrm{~d}$ & $2,80 \mathrm{a}$ & $3,75 \mathrm{a}$ & $2,82 \mathrm{c}$ & $0,71 \mathrm{a}$ & $14,64 \mathrm{~b}$ & $0,13 a$ \\
\hline & $7 \mathrm{mo}$ & $137,29 \mathrm{e}$ & 2,89 a & 4,22 c & $2,58 \mathrm{~b}$ & $0,74 \mathrm{a}$ & $19,38 d$ & $0,32 b$ \\
\hline & $8 \mathrm{vo}$ & $170,17 \mathrm{f}$ & $3,36 b$ & $4,06 \mathrm{~b}$ & $2,87 \mathrm{c}$ & $0,72 \mathrm{a}$ & $19,68 d$ & $0,54 \mathrm{c}$ \\
\hline & 9 no & $246,92 \mathrm{~g}$ & $3,55 b$ & $4,09 \mathrm{~b}$ & $2,75 \mathrm{c}$ & $0,73 \mathrm{a}$ & $18,12 \mathrm{c}$ & \\
\hline \multirow{8}{*}{ Portainjerto } & SL64 & $206,58 \mathrm{f}$ & & $4,46 \mathrm{e}$ & $3,21 \mathrm{f}$ & $1,00 \mathrm{f}$ & $24,11 \mathrm{~g}$ & $0,01 \mathrm{a}$ \\
\hline & MxM60 & 165,64 e & & $4,42 \mathrm{e}$ & $3,14 f$ & 0,92 e & $22,95 f$ & $0 \mathrm{a}$ \\
\hline & MxM14 & $111,14 \mathrm{~d}$ & & $4,29 d$ & $2,62 d$ & $0,76 d$ & $18,51 \mathrm{~d}$ & $0,01 \mathrm{a}$ \\
\hline & CAB6P & $94,33 \mathrm{c}$ & & 4,44 e & $2,72 d$ & $0,73 d$ & 20,32 e & $0,33 \mathrm{~b}$ \\
\hline & W13 & $85,86 \mathrm{c}$ & & $3,99 \mathrm{c}$ & $2,49 \mathrm{c}$ & $0,66 \mathrm{c}$ & 15,82 c & $0,57 \mathrm{c}$ \\
\hline & G5 & 28,72 a & & $2,42 \mathrm{a}$ & $1,63 \mathrm{a}$ & 0,32 a & 4,75 a & $0,01 \mathrm{a}$ \\
\hline & G6 & 49,6 b & & $3,16 \mathrm{~b}$ & $1,90 \mathrm{~b}$ & $0,45 b$ & $8,82 \mathrm{~b}$ & $0,00 \mathrm{a}$ \\
\hline & Adara-M & $201,68 \mathrm{f}$ & & $4,26 \mathrm{~d}$ & $3,02 \mathrm{e}$ & 0,94 e & $19,18 d$ & $1,17 \mathrm{~d}$ \\
\hline \multirow{2}{*}{ Variedad } & Bing & $122,49 \mathrm{~b}$ & 2,37 a & $4,25 b$ & $2,71 b$ & $0,77 \mathrm{~b}$ & 19,22 b & $0,36 \mathrm{~b}$ \\
\hline & Celeste & $113,40 \mathrm{a}$ & $3,68 \mathrm{~b}$ & $3,61 \mathrm{a}$ & $2,48 \mathrm{a}$ & $0,67 \mathrm{a}$ & $14,39 \mathrm{a}$ & $0,17 \mathrm{a}$ \\
\hline \multirow{16}{*}{$\begin{array}{l}\text { Portainjerto } \\
\text { variedad }\end{array}$} & B-SL64 & $180,15 \mathrm{~g}$ & & 4,63 h & $3,17 \mathrm{~g}$ & 0,98 j & 25,28 i & $0,02 \mathrm{a}$ \\
\hline & B-MxM60 & $174,13 \mathrm{~g}$ & & 4,64 h & $3,23 \mathrm{~g}$ & $0,97 \mathrm{j}$ & $25,31 \mathrm{i}$ & $0,00 \mathrm{a}$ \\
\hline & B-MxM14 & 119,98 e & & $4,51 \mathrm{~h}$ & 2,62 e & $0,80 \mathrm{~h}$ & $20,53 \mathrm{~g}$ & $0,01 a$ \\
\hline & B-CAB6P & $95,15 \mathrm{~d}$ & & $4,60 \mathrm{~h}$ & 2,73 e & $0,75 \mathrm{~g}$ & $21,70 \mathrm{~g}$ & $0,39 \mathrm{~b}$ \\
\hline & B-W13 & $97,31 \mathrm{~d}$ & & $4,38 \mathrm{~g}$ & $2,56 \mathrm{e}$ & $0,73 \mathrm{f}$ & $18,53 \mathrm{f}$ & $0,79 \mathrm{c}$ \\
\hline & B-G5 & 39,42 b & & $2,94 \mathrm{c}$ & $2,14 \mathrm{c}$ & $0,41 \mathrm{c}$ & $7,29 \mathrm{c}$ & $0,01 \mathrm{a}$ \\
\hline & B-G6 & $67,30 \mathrm{c}$ & & $3,68 d$ & $2,14 \mathrm{c}$ & $0,56 \mathrm{~d}$ & $12,11 d$ & $0,00 \mathrm{a}$ \\
\hline & B-AdaraM & $206,71 \mathrm{~h}$ & & $4,65 h$ & $3,09 \mathrm{f}$ & $1,00 \mathrm{~h}$ & $23,03 \mathrm{~h}$ & $1,63 d$ \\
\hline & C-SL64 & 233,01 i & & $4,29 \mathrm{~g}$ & $3,25 \mathrm{~g}$ & 1,02 h & $22,94 \mathrm{~h}$ & $0,00 \mathrm{a}$ \\
\hline & C-MxM60 & $157,14 \mathrm{f}$ & & $4,19 \mathrm{~g}$ & $3,06 \mathrm{f}$ & $0,86 \mathrm{~g}$ & $20,59 \mathrm{~g}$ & $0,00 \mathrm{a}$ \\
\hline & C-MxM14 & $102,30 \mathrm{~d}$ & & $4,08 \mathrm{f}$ & $2,63 \mathrm{e}$ & 0,71 e & 16,50 e & $0,00 \mathrm{a}$ \\
\hline & C-CAB6P & $93,51 \mathrm{~d}$ & & $4,28 \mathrm{~g}$ & $2,71 \mathrm{e}$ & 0,71 e & $18,94 \mathrm{f}$ & $0,28 \mathrm{~b}$ \\
\hline & C-W13 & 74,42 c & & $3,06 d$ & $2,42 d$ & $0,59 d$ & $13,11 d$ & $0,36 \mathrm{~b}$ \\
\hline & C-G5 & $18,02 \mathrm{a}$ & & 1,91 a & $1,12 \mathrm{a}$ & $0,24 \mathrm{a}$ & $2,21 \mathrm{a}$ & $0,00 \mathrm{a}$ \\
\hline & C-G6 & 32,17 b & & 2,63 b & $1,66 \mathrm{~b}$ & 0,35 b & 5,53 b & $0,00 \mathrm{a}$ \\
\hline & C-AdaraM & $196,60 \mathrm{~h}$ & & 3,88 e & $2,96 \mathrm{f}$ & $0,89 \mathrm{~g}$ & 15,33 e & $0,70 \mathrm{c}$ \\
\hline
\end{tabular}

Para cada columna y efecto, las medias seguidas por diferentes letras son significativamente diferentes según el Test de Scott \& Knott $(P<0,05)$.

${ }^{1}$ ADI (Años Después del Injerto), ${ }^{2}$ AST (Área Sección Transversal), ${ }^{3}$ RAST (Relación AST injerto-filtro) ${ }^{4}$ APD-H (Relación Área Proyección de Dosel y Altura), ${ }^{5} \mathrm{~V}$ (Índice de Vigor), ${ }^{6} \mathrm{VDct}$ Volumen de Dosel) y ${ }^{7} \mathrm{DS}$ (Densidad de sierpes).

Los portainjertos afectaron el AST $(P \leq 0,0001)$, diferenciándose seis grupos (Tabla 1). Los de mayor AST fueron el SL64 y M2624-Adara con incrementos sobre la media de $75 \%$ y $71 \%$ respectivamente (De Salvador et al., 2005), seguido por MxM60 con un $40 \%$ de incremento sobre la media y luego el MxM14 con una reducción del 6\% de la media. Un cuarto grupo, formado por los guindos, CAB6P y W13 con una reducción de 20\% y $27 \%$ de la media, respectivamente (De Salvador et al., 2005; Stehr, 2001; López-Ortega et al., 2016). Cada GiSeLa constituyó un grupo; el G6 con una reducción del $58 \%$ y el G5 con una del $76 \%$ de la media (De Salvador et al., 2005; Sitarek et al., 2005; Whiting et al., 2005; Sansavini y Lugli, 2014). Se destaca la importancia de las condiciones locales sobre las características de los portainjertos. Santos et al. (2006) sugirieron que los doseles de MxM14 y G5 no son diferentes, mientras que Lichiev \& Papachatzis (2009) encontraron que W13 tenía diferencias mínimas con P. mahaleb L.

La variedad afectó $(P \leq 0,0001)$ el AST de Celeste, que fue un $92,6 \%$ del de Bing (Tabla 1) (Usenik et al., 2006). 
Lacombinación portainjerto-variedad $(P \leq 0,0001)$ influyó en las características del árbol (Tabla 1). Tanto CAB6P como Adara-M2624 mostraron un AST que no diferenció entre variedades. El SL64 mostró un AST 29,3\% mayor con Celeste que con Bing. En el resto de los portainjertos el AST fue mayor con Bing. La combinación G5-Celeste fue la más restrictiva, con un 15\% del promedio, seguido por G5-Bing y G6-Celeste con una reducción de $33 \%$ y $27 \%$ respectivamente (Long \& Kaiser, 2010; Sansavini \& Lugli, 2014). Los portainjertos W13, G6 y G5 mostraron una reducción del AST cuando fueron injertados con Celeste de 24\%, 52\% y 54\%, respecto de Bing. Mientras que MxM60, MxM14, CAB6P y Adara-M2624 mostraron reducciones de $10 \%, 15 \%, 2 \%$ y $5 \%$ respectivamente (Tabla 1 ). Ello sugiere que los portainjertos menos vigorosos son más influenciados por el vigor de la variedad. Esta interacción es la causa de que cada combinación posea características ligeramente propias (Atkinson y Else, 2001; Usenik et al., 2006).

La interacción bloque-portainjerto (Figura 1) mostró que los más vigorosos, como Adara-M2624, SL64 y MxM60, tuvieron buen comportamiento en suelos con ligeras limitaciones de salinidad (bloque II). Se destaca G5 por su comportamiento estable entre bloques.

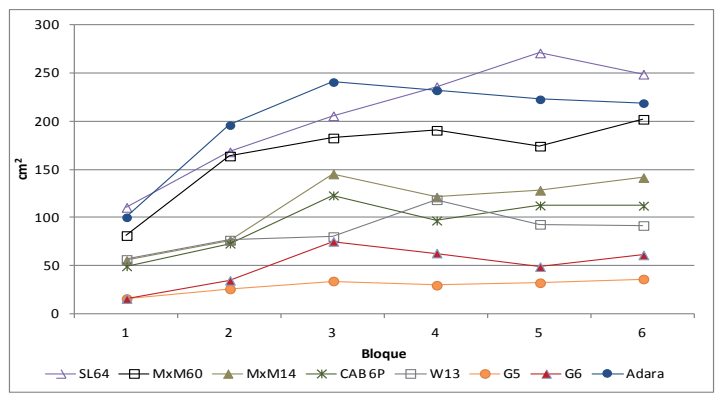

Figura 1. Efecto interacción bloque-portainjerto sobre el AST.

La interacción ADI-portainjerto muestra que tanto los Adara-M2624 y SL64 como los CAB6P y W13 poseen comportamientos semejantes. El noveno ADI (2013) se caracterizó por la ausencia de carga frutal, en consecuencia, el AST experimentó mayores incrementos que variaron con el portainjerto. Tanto MxM60, como SL64 aumentaron el AST, respecto del octavo ADI, $54 \%$ y $48 \%$, respectivamente, mientras que G5 sólo 39\% (Figura 2). Ello sugiere que G5 induce un bajo crecimiento vegetativo, aun en condiciones de ausencia de carga.

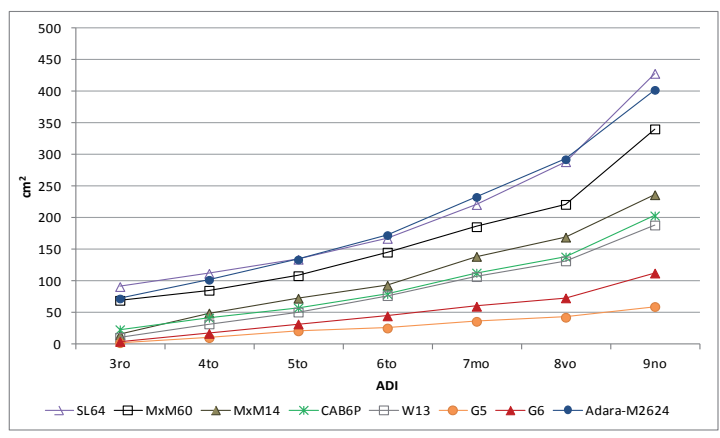

Figura 2. Efecto interacción edad-portainjerto sobre el AST.

\section{Relación área de sección transversal del tronco de la combinación $P$. avium-Adara}

La RAST media indicó que el AST de P. avium fue 3,01 veces mayor que el AST del filtro Adara con una $\mathrm{DE} \pm 0,9$. El modelo indicó que la RAST estuvo influenciada $\left(R^{2}=0,81 ; P \leq 0,0001\right)$ por la edad $(P \leq 0,0001)$ y la variedad $(P \leq 0,0001)$. El mayor poder explicativo lo tuvo la variedad (54\%); luego, la edad (18\%).

La RAST se incrementó con la edad. Al quinto $A D I$, la RAST fue de 2,53 veces, mientras que al noveno ADI la RAST fue 3,55 veces, un $40 \%$ mayor. Esto indica que, con el tiempo, la diferencia de AST entre filtro y variedad aumenta (Tabla 1). La variedad tiene fuerte influencia en la RAST; Bing mostró una RAST de 2,37 veces, mientras que Celeste fue 3,68 veces, un $55 \%$ mayor (tabla 1). Se ha sugerido que los filtros pueden afectar el tamaño y la longevidad de los cerezos (Rozpara et al., 1998; Magyar \& Hrotko, 2008) y, además, que portainjertos de ciruelo pueden reducir el vigor (De Salvador et al., 2005; Sitarek et al., 2005; Gonçalves et al., 2007; Jiménez et al., 2007). Aunque Adara utilizado como portainjerto de cerezo presenta buena compatibilidad (Moreno et al., 1995; Moreno et al., 1996), su uso como filtro tiene pocos antecedentes (López-Ortega et al., 2016). La elevada RAST es causada por una severa hiperplasia que se corresponde a altas concentraciones de hidratos de carbono por encima de la unión injerto-filtro (Cohen et al., 2007; Olmstead et al., 2010) y bajas concentraciones en el portainjerto (Yano et al, 2002) que pueden, eventualmente, interferir con el transporte de agua, nutrientes y fotosintatos, e incrementar la resistencia hidráulica (Errea et al, 1994; Olmstead et al, 2010). Por lo tanto, un alto RAST puede implicar cierta restricción del crecimiento (Deloire \& Hebant, 1983; Franken-Bembenek, 1996; Hrotkó, 1998; Yano, 2002; Pina \& Errea, 2005; Olmstead et al, 2010). Además de la hiperplasia, la variedad Celeste mostró ligeros síntomas de epinastia que 
sugiere cierto grado de incompatibilidad localizada (Hrotkó, 2008, Sansavini y Lugli, 2014), e indica la necesidad de realizar estudios complementarios.

\section{Altura}

La $\mathrm{H}$ media fue $3,93 \mathrm{~m}$ con una $\mathrm{DE} \pm 0,95$. La $\mathrm{H}$ mínima fue $0,5 \mathrm{~m}$ y la máxima fue $5,45 \mathrm{~m}$. La H estuvo correlacionada al AST $(r=0,70 ; P \leq 0,0001)$ similar a lo observado por Moreno et al. (2001) y además, relacionada a través de un modelo lineal con el LN del AST $\left(R^{2}=0,74 ; P \leq 0,0001\right)$.

La variabilidad de $H$ fue explicada $\left(R^{2}=0,91\right.$; $P \leq 0,0001)$ por los efectos bloque $(P \leq 0,0001)$, ADI $(P \leq 0,0001)$, portainjerto $(P \leq 0,0001)$, variedad $(P \leq 0,0001)$, interacción portainjerto-variedad $(P \leq 0,0001)$ y bloque-portainjerto $(P \leq 0,0001)$. Los efectos de mayor poder explicativo fueron portainjerto $(54 \%)$ y variedad $(11,6 \%)$.

La $\mathrm{H}$ media anual se incrementó con la edad. No se observaron diferencias entre el quinto y el sexto ADI, pero sí entre el séptimo y el octavo. Durante el invierno del séptimo ADI (2011) se realizó una poda de control de $\mathrm{H}$ que la redujo en los años siguientes, independientemente del portainjerto y variedad (Tabla 1).

Los antecedentes indican que los portainjertos influyen en la altura de las plantas (Moreno et al., 2001; Santos et al., 2006). La Tabla 1 muestra que los portainjertos que indujeron doseles más altos fueron SL64, MxM60 y CAB 6P, que alcanzaron respectivamente $99 \%$, 98\% y 99\% de la H máxima, seguidos por MxM14 y Adara-M2624, ambos con el 95\% de la H máxima. El W13 con 3,99 m tuvo $89 \%$ de la $\mathrm{H}$ disponible. Los más bajos fueron G6 con 3,16 m y G5 con 2,42 m que correspondieron a $70 \%$ y $54 \%$ de la $\mathrm{H}$ máxima, respectivamente (Prassinos et al., 2009).

La H de Bing fue un 15\% mayor que la de Celeste. La interacción portainjerto-variedad mostró que Celeste sobre Adara-M2624, W13, G6 y G5 indujo una reducción de la $\mathrm{H}$ correspondiente a $20 \%$, $22 \%, 40 \%$ y $54 \%$, respecto de Bing; mientras que con MxM14, CAB6P, SL64 y MxM60 las respectivas caídas fueron de $11 \%, 7 \%, 8 \%$ y $11 \%$ (Tabla 1 ). Ello indica que la $\mathrm{H}$ inducida por los portainjertos Adara-M2624, W13, G6 y G5 varía más entre Bing y Celeste que con MxM14, CAB6P, SL64 y MxM60 (Atkinson y Else, 2001; Usenik et al., 2006).

Las combinaciones que superaron la $\mathrm{H}$ disponible, aun con intervención de poda, fueron: Bing sobre SL64, MxM60, CAB6P y Adara-M2624 (Tabla 1). Esto sugiere que el control de la $\mathrm{H}$ mediante poda en combinaciones de variedades y portain- jertos de vigor medio o alto presenta dificultades.

La interacción bloque-portainjerto (Figura 3) muestra que las condiciones edáficas de los primeros bloques redujeron la $\mathrm{H}$ de todos los portainjertos. Se destaca que el MxM60, alcanzó la H disponible en las condiciones limitantes del bloque II.

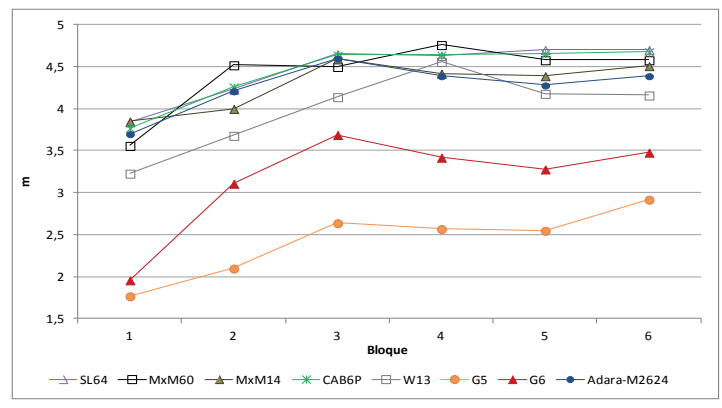

Figura 3. Efecto interacción bloque-portainjerto sobre la $\mathrm{H}$. Test de Scott \& Knott $(\alpha=0,05)$.

\section{Relación área de proyección del dosel-altura}

La relación APD-H media fue 2,59 $\mathrm{m}^{2} \mathrm{~m}^{-1}$. El modelo $\left(R^{2}=0,85 ; P \leq 0,0001\right)$ mostró que la relación fue influenciada por los $A D I(P \leq 0,0001)$, bloque $(P \leq 0,0001)$, portainjerto $(P \leq 0,0001)$, variedad $(P \leq 0,0001)$, interacción portainjerto-variedad $(P \leq 0,0001)$ y variedad-bloque $(P \leq 0,0001)$. La mayor variabilidad fue explicada por portainjerto (42\%) y ADI (18\%). La edad influyó sobre la APD$\mathrm{H}$, lo que indica que la forma de la planta evoluciona, tendiendo a ser más globosa (Tabla 1). Aunque en el séptimo ADI se observa una reducción del APD-H como consecuencia del incremento de $\mathrm{H}$, en los años siguientes se incrementa debido al control de $\mathrm{H}$ con poda.

Los portainjertos que indujeron doseles más globosos fueron SL64, MxM60 y algo menos el AdaraM2624, mientras que los doseles de MxM14, CAB6P y W13 tuvieron formas alargadas. Los G6 y G5 indujeron doseles muy alargados, contrariamente a lo observado por Sitarek et al. (2005) en árboles no podados. La poda influye en la arquitectura del dosel (Lauri \& Claverie, 2008) y si bien, las ramas laterales tienden a ser más horizontales y mejor iluminadas (Sitarek et al., 2005), la poda las acorta y reduce el ancho del dosel. El dosel de la variedad Celeste tiende a ser más alargado que el de Bing. Los G5, G6 y W13 inducen doseles más globosos con Bing que con Celeste (Tabla 1). La interacción variedad-bloque mostró que el dosel de Celeste en suelos menos fértiles es más alargado, pero a medida que mejora la fertilidad, tiende a ser más globoso. Esto sugiere que en suelos fértiles la forma de los doseles tiende a asemejarse (Figura 7). 


\section{Volumen de dosel cono truncado}

El VD $D_{\text {ct }}$ medio fue $16,8 \mathrm{~m}^{3}$ con una $\mathrm{DE} \pm 8,9 \mathrm{~m}^{3}$, un mínimo de 0,04 $\mathrm{m}^{3}$ y un máximo de $43,3 \mathrm{~m}^{3}$. El $V D_{c t}$ estuvo fuertemente correlacionado con: AST $(r=0,82 ; P \leq 0.0001), H(r=0,92 ; P \leq 0.0001)$ y APD-H $(r=0,84 ; P \leq 0,0001)$ que es consistente con lo observado por Moreno et al., (2001).

El LN del AST se relacionó con el $V D_{c t}$ mediante un modelo lineal $\left(R^{2}=0,71 ; P \leq 0,0001\right)$, semejante a lo observado por Smith (2008). Pero, al incorporar la información de los portainjertos, el modelo LNAST-VD ${ }_{\text {ct }}$-portainjerto mejoró la calidad del ajuste $\left(R^{2}=0,79 ; P \leq 0,0001\right)$. El modelo AST-VDct fue sometido a una prueba de comparación de regresiones mediante el método de modelos anidados, a fin de estudiar si explica adecuadamente las diferencias que eventualmente pudiesen existir entre portainjertos. Las disparidades pueden deberse al valor inicial de la relación, a la tasa o a ambos. Los resultados indican que la información aportada tanto, por las variables que representan la relación AST-VD ${ }_{c t}$ de los portainjertos al inicio, como las de las tasas, mejoran la calidad de ajuste del modelo $(P \leq 0,005)$. Por lo cual, hay portainjertos o grupo de ellos, cuya relación AST-VDct es significativamente diferente. Si bien, todos los portainjertos se relacionaron con el mismo modelo, cada portainjerto o grupo de ellos tuvo sus propios parámetros. La Figura 4 muestra que para alcanzar el VD $D_{c t}$ máximo disponible $\left(20,78 \mathrm{~m}^{3}\right)$ el CAB6P necesitó un AST del injerto de $106 \mathrm{~cm}^{2}$ y, por lo tanto, la relación entre el VD $D_{c t}$ y el AST fue 0,196 $\mathrm{m}^{3}$ por cada $\mathrm{cm}^{2}$. Con SL64, MxM60 o MxM14 se necesitaron 144 $\mathrm{cm}^{2}$ para alcanzar el dosel máximo, es decir 0,144 $\mathrm{m}^{3} \mathrm{~cm}^{-2}$. Con $\mathrm{G} 6$ se requirió $164 \mathrm{~cm}^{2}$ ó $0,126 \mathrm{~m}^{3}$ $\mathrm{cm}^{-2}$, con W13 fueron $184 \mathrm{~cm}^{2}$ ó $0,113 \mathrm{~m}^{3} \mathrm{~cm}^{-2} \mathrm{y}$ con Adara-M2624, $273 \mathrm{~cm}^{2}$ ó 0,077 $\mathrm{m}^{3} \mathrm{~cm}^{-2}$. G5 no alcanzó el $V D_{c t}$ máximo disponible, ya que midió $110 \mathrm{~cm}^{2}$ de AST y un $V D_{\text {ct }}$ de $9,77 \mathrm{~m}^{3}$ con una relación $0,089 \mathrm{~m}^{3} \mathrm{~cm}^{-2}$ ). Consecuentemente, aunque ambas variables son indicadoras de vigor, la evidencia sugiere que el AST permite comparar sólo los efectos producidos por diferentes condiciones en un mismo portainjerto, pero muestra limitaciones al comparar diferentes portainjertos para una misma condición. Por lo tanto, es posible que AST y $V D_{c t}$ clasifiquen de un modo diferente a un mismo portainjerto; además, ello indica que la relación VD $D_{c t}$-AST no influye en el vigor de la planta. Tanto G5 como Adara-M2624 muestran una baja relación $V D_{c t}-A S T$ con VD ct muy diferentes, mientras que la relación $\mathrm{VD}_{\mathrm{ct}}$-AST de G5 es la mitad de la de CA$\mathrm{B} 6 \mathrm{P}$ con un dosel cuatro veces menor.

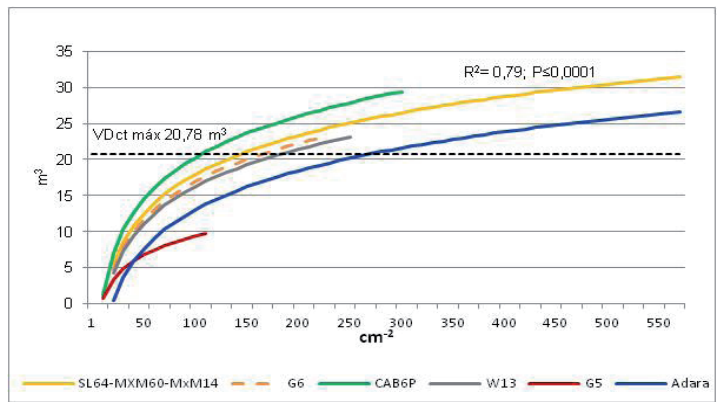

Figura 4. Efecto del portainjerto sobre la relación entre el AST y el VDct.

El modelo diferencia el comportamiento de ocho portainjertos según la prueba de $\mathrm{F}$ de comparación de modelos anidados $(\mathrm{P} \leq 0,005)$.

La variabilidad del $V D_{\text {ct }}$ fue explicada $\left(R^{2}=0,91\right.$; $P \leq 0,0001)$ por los efectos bloque $(P \leq 0,0001)$, ADI $(P \leq 0,0001)$, portainjerto $(P \leq 0,0001)$, variedad $(P \leq 0,0001)$, interacción portainjerto-variedad $(P \leq 0,0001)$, bloque-portainjerto $(P \leq 0,0001)$ y $A D I-$ portainjerto $(P \leq 0,0001)$. Los efectos de mayor poder explicativo fueron portainjerto (51\%) y ADI (11\%).

$E l D_{c t}$ se incrementó anualmente alcanzando cierta estabilidad en el séptimo ADI con aproximadamente el $95 \%$ del $V D_{c t}$ máximo disponible. El $V D_{c t}$ en el noveno ADI se redujo como consecuencia de la poda (Tabla 1).

EI VD fue influido por el portainjerto. El SL64 y el MxM60 superaron $16 \%$ y $10 \%$ del VD disponible, respectivamente. El CAB6P alcanzó el 98\% del VD disponible, seguido por el Adara-M2624 y MxM14 con $92 \%$ y $89 \%$, respectivamente. Mientras que W13, G6 y G5 se diferenciaron entre sí, ocupando $76 \%, 42 \%$ y $23 \%$, respectivamente del VD disponible (Tabla 1), consistente con lo observado por Stehr (2001), De Salvador et al., (2005), Sitarek et al., (2005) y Whiting et al., (2005). La variedad Celeste indujo una reducción del $25 \%$ respecto de Bing (Tabla 1). Se observa que Adara-M2624, al presentar hiperplasia, tiene una mayor AST (Jiménez et al., 2007), con menor $V D_{c t}$ que CAB6P y este a su vez presenta menor AST y mayor VD ${ }_{\text {ct }}$ que MxM14 (Gratacós et al., 2007). Tales inconsistencias se explican porque $C A B 6 P$ posee mayor $V_{\text {ct }}$ por unidad de AST en relación a Adara-M2624 y MxM14.

Para un mismo portainjerto, el VD ct varió cuando se cambió la variedad, aunque algunos mostraron mayores diferencias que otros. Los portainjertos más uniformes al cambio varietal fueron SL64, MxM60, MxM14 y CAB6P, que mostraron diferencias de $10 \%, 23 \%, 24 \%$ y $15 \%$ respectivamente entre Bing y Celeste. A su vez, el cambio varietal en G5 o G6 indujo diferencias de $230 \%$ y $119 \%$, respectivamen- 
te entre Bing y Celeste. El W13 y el Adara-M2624 mostraron valores intermedios con variaciones del $V D_{\text {ct }}$ de $41 \%$ y $50 \%$, respectivamente (Tabla 1 ). Los doseles que superaron el $V D_{c t}$ máximo disponible correspondieron a las combinaciones injerto-portainjerto: Bing o Celeste sobre SL64 y Bing sobre MxM60, Adara-M2624 o CAB6P (Tabla 1).

$E I V D_{c t}$ de los portainjertos varió fuertemente con la fertilidad de los bloques (Figura 5). La interacción bloque-portainjerto mostró que el $V D_{c t}$ de G5 tuvo un comportamiento más uniforme que G6. El SL64 mostró un buen desempeño en las condiciones de salinidad ligera del bloque II, mientras que MxM60 superó el volumen disponible. Ambos GiSeLa mantuvieron el VD ct por debajo del disponible (Sansavini \& Lugli, 2014; López-Ortega et al., 2016) al igual que W13 (Stehr., 2001). El VD ct de MxM14 y Adara-M2624 pudo ser controlado mediante poda sólo en algunos bloques.

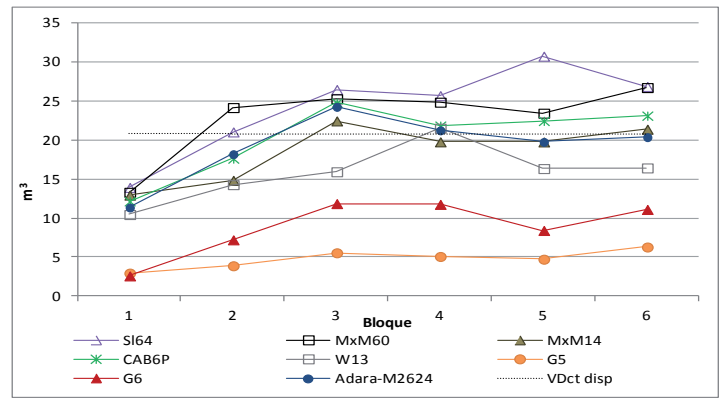

Figura 5. Interacción bloque-portainjerto sobre el VDct. Test de Scott \& Knott $(\alpha=0,05)$.

La Figura 6 muestra que el $V D_{\text {ct }}$ inducido por W13, G6 y G5 estuvo siempre por debajo del VD disponible. Mientras que, a partir del séptimo ADI, el VDct del SL64, MxM60 y CAB6P no fue controlado en las condiciones del ensayo y el de AdaraM2624 y MxM14 pudo ser controlado mediante poda al noveno ADI.

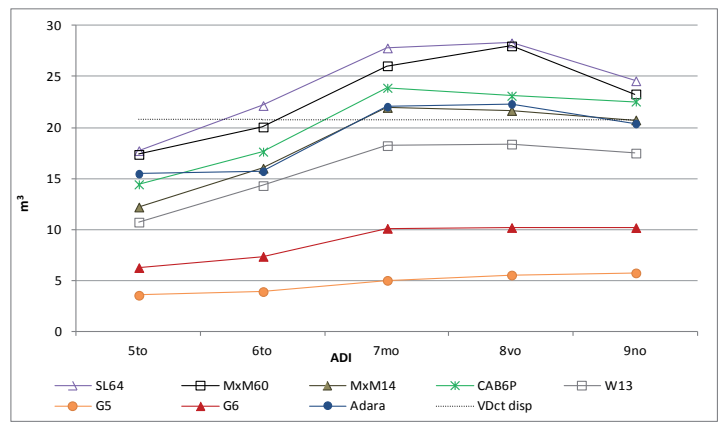

Figura 6. Interacción edad-portainjerto sobre el VDct. Test de Scott \& Knott $(\alpha=0,05)$.
Los antecedentes indican que Adara, como portainjerto autoenraizado, es más vigoroso que SL64 (Moreno et al., 1996; Jiménez et al., 2007; Cantín et al., 2010). Sin embargo, los resultados muestran que el $V D_{c t}$ inducido por Adara como filtro sobre M2624 es 20\% menor que el de SL64. Rozpara et al. (1998) encontraron que tanto el filtro como la interacción filtro-portainjerto inducen reducción del vigor. Los resultados de este ensayo son consistentes con estos datos. El uso de filtros de ciruelo intercompatibles con cerezos es novedoso y de gran potencial, y aún ha sido poco estudiado. El vigor intermedio combinado con la tolerancia a la hipoxia del M2624 puede ser recomendable en suelos pobres, anegadizos o bajo condiciones de replantes (Moreno, 2004; Cantin et al., 2010; Pimentela et al., 2014).

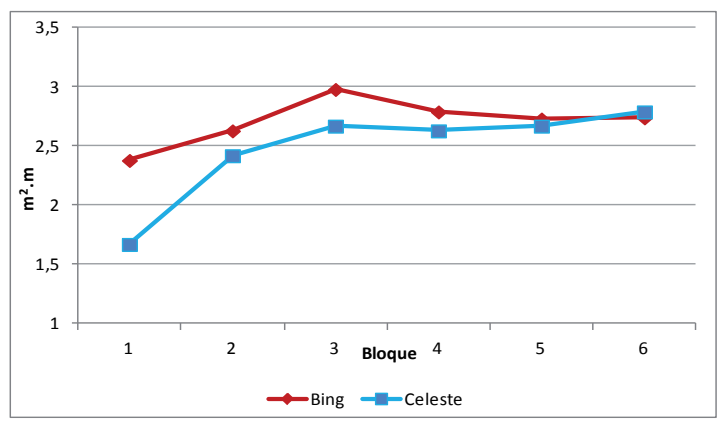

Figura 7. Interacción bloque-variedad sobre la APD-H. Test de Scott \& Knott $(\alpha=0,05)$.

\section{Índice de vigor}

El IV medio fue 0,26 y mostró una correlación positiva con AST ( $r=0,88 ; P \leq 0,0001)$, con $H(r=0,84$; $P \leq 0,0001)$ y con $V D c t(r=0,92 ; P \leq 0,0001)$.

El modelo $\left(R^{2}=0,93 ; P \leq 0,0001\right)$ mostró que la variabilidad de este índice fue explicada por los efectos bloque $(P \leq 0,0001)$, portainjerto $(P \leq 0,0001)$, variedad $(P \leq 0,0001)$ e interacción portainjertovariedad $(P \leq 0,0001)$. El mayor poder explicativo correspondió a portainjerto (65\%).

El mayor IV correspondió a SL64, seguido por MxM60 y M2624-Adara con 0,92 y 0,94 respectivamente; un grupo intermedio formado por MxM14 con 0,76 y CAB6P con 0,73. Mientras que W13, G6 y G5 se diferenciaron entre sí con IV de 0,66; 0,45 y 0,32 respectivamente (Tabla 1 ).

El IV de la variedad Celeste fue el $87 \%$ del de Bing (Tabla 1).

Los portainjertos SL64 y CAB6P mostraron uniformidad de dosel al cambio varietal, mientras que 
el resto mostró mayor variación. El portainjerto G5 redujo el IV de $41 \%$ a $24 \%$ cuando fue injertado con Bing o Celeste, respectivamente. Mientras que el IV de G6 se redujo de 56\% (Tabla 1) a 25\%, respectivamente. La buena correlación entre el IV con el resto de los indicadores así como el alto poder explicativo del modelo, confirman las bondades de este índice empírico propuesto por Stehr (2001).

\section{Densidad de sierpes}

El promedio de DS fue $0,26 \mathrm{~S} \mathrm{~m}^{-2}$, con una $\mathrm{DE} \pm 0,73$ y un $C V$ de $277 \%$. El valor mínimo y máximo fue 0 y $5,58 \mathrm{~S} \mathrm{~m}^{-2}$, respectivamente. El modelo $\left(R^{2}=0,87 ; P \leq 0,0001\right)$ mostró que la variabilidad de la DS fue explicada por bloque $(P \leq 0,0001)$, $A D I(P \leq 0,0001)$, portainjerto $(P \leq 0,0001)$, variedad $(P \leq 0,0001)$ e interacciones portainjerto-variedad $(P \leq 0,0001)$, bloque-portainjerto $(P \leq 0,0001)$ y $A D I-$ portainjerto $(P \leq 0,0001)$. Los efectos con mayor poder explicativo fueron: portainjerto $(30 \%)$ y su interacción con ADI (18\%).

La DS estuvo asociada a la edad de las plantas, con un incremento sostenido, pasando de 0,07 S $\mathrm{m}^{-2}$ en el quinto ADI a 0,54 $\mathrm{S} \mathrm{m}^{-2}$ al octavo ADI (Tabla 1). Menzies (1998) menciona un incremento de los sierpes en Prunus cerasus var. Stockton Morello desde la plantación con un máximo al tercer año. Los portainjertos que formaron sierpes fueron los guindos CAB6P (De Salvador et al., 2005; Santos et al., 2006) y W13 (Lichiev \& Papachatzis, 2009) y el ciruelo M2624. El portainjerto M2624 presentó una media de $1,17 \mathrm{~S} \mathrm{~m}^{-2}$, produciendo más del doble que W13 y cuatro veces más que CAB6P; ambos son considerados portainjertos con fuerte tendencia a la formación de sierpes. El resto tuvo nula o casi nula formación (Tabla 1) (Atkinson y Else, 2001; Lichiev \& Papachatzis, 2009). La variedad Bing indujo el doble de DS que Celeste (Tabla 1).

Los portainjertos W13 y Adara-M2624 mostraron el doble de DS cuando fueron injertados con Bing respecto de Celeste (Tabla 1).

La Figura 8 muestra que la DS de CAB6P es más uniforme entre bloques que la de W13 y M2624. A partir del bloque II, la DS de W13 tiende a reducirse, mientras que la de M2624 se incrementa hasta el bloque $\mathrm{V}$ concomitante con el máximo contenido de $L+A$.

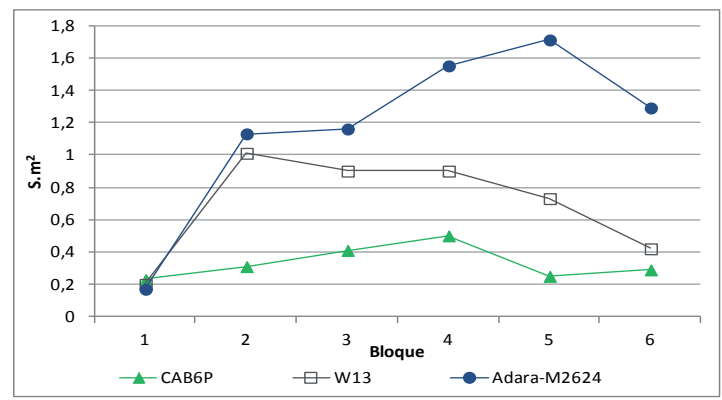

Figura 8. Interacción bloque-portainjerto sobre la DS. Test de Scott \& Knott $(\alpha=0,05)$

La Figura 9 muestra que la DS de CAB6P es relativamente baja y estable entre ADI, mientras que la de W13 se incrementa del séptimo al octavo ADI, y la de Adara-M2624 es la mayor desde el inicio.

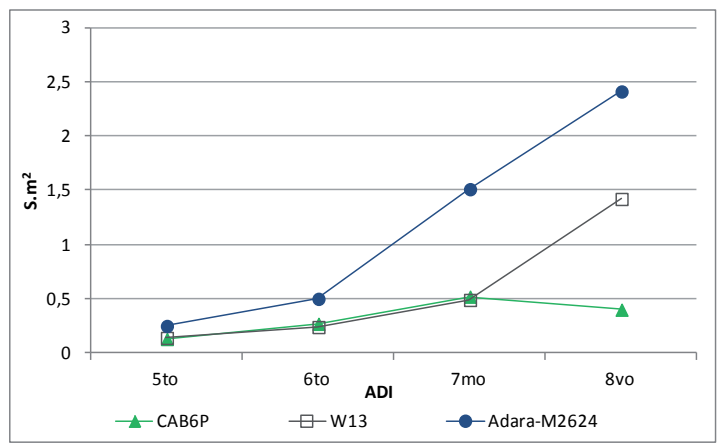

Figura 9. Interacción edad-portainjerto sobre la DS. Test de Scott $\&$ Knott $(\alpha=0,05)$.

La elevada DS de la combinación Adara-M2624 indica la necesidad de hallar un portainjerto sin sierpes y de buena adaptación.

\section{CONCLUSIONES}

Los portainjertos mostraron una fuerte influencia sobre las características vegetativas del dosel y es el factor que mejor explica el control del tamaño de los árboles.

En las condiciones del ensayo, el portainjerto SL64 tuvo un comportamiento muy vigoroso ya que presenta los indicadores más altos. Fue seguido por MxM60, considerado vigoroso por mostrar AST e IV significativamente más bajos. Tanto MxM14 y CAB6P como Adara-M2624 fueron semivigorosos, por presentar valores intermedios en sus indicadores. Se destacó la combinación Adara-M2624 que mostró una fuerte hiperplasia que se acentuó con el tiempo. El W13 mostró un comportamiento semienanizante, con indicadores más bajos, aunque 
el AST fue semejante al de CAB6P. Ambos Gisela fueron los más enanizantes, aunque, G6 mostró mayor vigor que G5, que fue considerado muy enanizante por presentar los indicadores más bajos.

La influencia de la variedad sobre el vigor del árbol fue menor que la del portainjerto. La combinación de un portainjerto muy enanizante con una variedad de escaso vigor indujo árboles demasiado débiles; la situación inversa produce plantas muy vigorosas. La formación de sierpes de M2624 fue mayor que la de CAB6P y W13.

Debido a las cualidades observadas en AdaraM2624 se sugiere continuar investigando con portainjertos adaptados a condiciones de suelo pesado, pero sin formar hiperplasias o sierpes.

\section{AGRADECIMIENTOS}

Los autores agradecen a los viveros Puertas Hnos, Agroviveros S.A. y Bobadilla S.A. por la valiosa y permanente colaboración recibida durante la ejecución del ensayo.

\section{BIBLIOGRAFÍA}

Atkinson, C. and M. Else, 2001. Understanding how rootstocks dwarf fruit tree. Compact Fruit Tree 34: 46-49.

Balmer, M., 2008. Evaluation of semi-dwarfing rootstocks for sweet cherry orchards in the Rhine River Valley (Germany). Acta Horticulturae 795: 203-207.

Battistini, A., 2005. A semi-dwarfing cherry rootstock for dry conditions. Acta Horticulturae 667: 189-190.

Cantín, C.; J. Pinochet, Y. Gogorcena and M. A. Moreno, 2010. Growth, yield and fruit quality of 'Van' and 'Stark Hardy Giant' sweet cherry cultivars as influenced by grafting on different rootstocks. Scientia Horticulturae 123: 329-335.

Charlot, G.; M. Edin, F. Flochlay, P. Soing and C. Boland, 2005. Tabel Edabriz: a dwarf rootstocks for intensive cherry orchards. Acta Horticulturae 667: 217-221.

Cohen, S.; A. Naor, J. Bennink, A. Grava and M. Tyree, 2007. Hydraulic resistance components of mature apple trees on rootstocks of different vigours. Journal of Experimental Botany, 58, (15-16): 4213-4224.

Deloire, A. and C. Hebant, 1983. Histophysiological study of the graft union of compatible and incompatible combinations of Prunus. Agronomie 2: 207-212.

De Salvador, F.; G. Di Tommaso, C. Puccioni. and P. Bonofiglio, 2005. Performance of new and standard cherry rootstocks in different soil and climatic conditions. Acta Horticulturae 667: 191-199.

Di Rienzo, J. A.; F. Casanoves, M. G. Balzarini, L. Gon- zalez, M. Tablada y C.W.Robledo, 2015. InfoStat versión 2015. Grupo InfoStat, FCA, Universidad Nacional de Córdoba, Argentina. <http://www.infostat.com.ar> (Consultada el 08/07/2014)

Errea P.; A. Felipe and M. Herrero, 1994. Graft establishment between compatible and incompatible Prunus spp. Journal Experimental Botany 45: 393-401.

Franken-Bembenek, S., 1996. The Giessen cherry rootstocks. Compact Fruit Tree 29: 19-36.

Franken-Bembenek, S., 2005. Gisela®5 rootstock in Germany. Acta Horticulturae 667: 167-172.

Gonçalves, B.; C. M. Correia, A., P. Silva, E. A. Bacelar, A. Santos, H. Ferreira and J. M. Moutinho-Pereira, 2007. Variation in xylem structure and function in roots and stems of scion-rootstock combinations of sweet cherry tree (Prunus avium L.). Trees 21:121-130.

González, M., 1992. Guía para el cálculo de las exigencias de fertilización de acuerdo a las disponibilidades de nitrógeno, fósforo y potasio del suelo. INTA Centro Regional Cuyo, EEA Mendoza. Folleto 107. 10 pp.

Gratacós, N.; B. Cortés and B. Kulczewski, 2007. New rootstocks for the Chilean cherry industry. Compact fruit tree 4:19-21.

Grzyb, Z. and M. Sitarek, 2005. Results of sweet cherry rootstock trial in Northen Poland. Acta Horticulturae 667: 207-216.

Guajardo, L., 2004. Comportamiento fenológico y productivo del cerezo (Prunus avium L.) cv. Lapins, sobre diferentes portainjertos y sistemas de conducción, en la comuna de Curicó. Tesis de Maestría. Pontificia Universidad Católica de Valparaíso, Chile. 85 pp.

http://cerfundao.pt/eng/documentos/Enxertos\%20-\%206. pdf (Consultada el 16/11/2016)

Gyeviki, M.; K. Hrotkó and P. Honfi, 2012. Comparison of leaf population of sweet cherry (Prunus avium L.) trees on different rootstocks. Scientia Horticulturae 141: 30-36.

Hilsendegen, P., 2005. Preliminary results of a National German Sweet Cherry Rootstock trial. Acta Horticulturae 667:179-187.

Hrotkó K.. 2008. Progress in cherry rootstock research. Acta Horticulturae 795:171-178

Hrotkó, K., L. Magyar and G. Simon, 1998. Growth and productivity of sweet cherry interstem trees. Acta Horticulturae 468:353-362

Jiménez S.; J. Pinochet, Y. Gogorcena, J. Betrán. and M. Moreno, 2007. Influence of different vigour cherry rootstocks on leaves and shoots mineral composition. Scientia Horticulturae 112, 73-79.

Kappel, F. and G. Lang, 2008. Performance of the NC-140 Regional sweet cherry rootstock trial planted in 1998 in North America. Acta Horticulturae 795: 317-320. 
Kappel, F.; G. Lang, L. Anderson, A. Azarenko, T. Facteau, A. Gaus and S. Southwick, 2005. NC-140 Regional cherry rootstocks trial (1998): results from western North America. Acta Horticulturae 667: 223-232.

Lauri, P. and J. Claverie, 2008. Sweet cherry tree architecture, physiology and management: towards an integrated view. Acta Horticulturae 795, p.605-614.

Lichiev, V. and A. Papachatzis, 2009. Results from the 11year evaluation of 10 rootstocks of the sweet cherry cultivar 'Stella'. Acta Horticulturae 825:513-520.

Long, L. and C. Kaiser, 2010. Sweet cherry roostocks for the Pacific Northwest. PNW619 A Pacific Northwest Extension Publication. Oregon State University, Washington State University, University of Idaho and Washington State University. 8 pp <http://extension.oregonstate.edu/wasco/sites/default/files/sweet_cherry_ rootstocks_pnw619.pdf > (Consultada el 16/11/2016).

López-Ortega, G.; F. García-Montiel, A. Bayo-Canhaa, C. Frutos-Ruiza and D. Frutos-Tomás, 2016. Rootstock effects on the growth, yield and fruit quality of sweet cherry cv. 'Newstar' in the growing conditions of the Region of Murcia. Scientia Horticulturae 198:326-335.

Magyar, L. and K. Hrotko, 2008. Prunus cerasus and Prunus fructicosa as interstocks for sweet cherry tree. Acta Horticulturae 795:287:292.

Mendenhall, W. and T. Sincich, 1995. Statistics for engineering and the sciences. 4a. edition. Prentice Hall, New Jersey. 787 pp.

Menzies, A. 1998. Year to year in productivity, nutrition and suckering of four cherry rootstocks during a 12 year evaluation. Acta Horticulturae 468:327-331

Moreno, M., 2004. Mejora y selección de patrones de frutales de hueso en la estación experimental Aula Dei. ITEA 99v 1: 11-22.

Moreno, M.; M. Tabuenca and R. Cambra, 1995. Adara, a plum rootstock for cherries and other stone fruit species. Hortscience 30 (6):1316-1317.

Moreno, M.; L. Montañés, M. Tabuenca and R. Cambra, 1996. The performance of Adara as a cherry rootstock. Scientia Horticulturae 65: 85-91.

Moreno, M.; R. Adrada, J. Aparicio and J. Betra, 2001. Performance of Sunburst sweet cherry grafted on different rootstocks. Journal of Horticultural Science and Biotechnology 2: 167-173.

Neilsen, G.; D. Neilsen, F. Kappel, P. Toivonen and L. Herbert, 2010. Factors affecting establishment of sweet cherry on Gisela 6 rootstock. Hortscience 45 (6): 939 945.

Olmstead, M.; N. Lang, G. Lang, F. Ewers, F. Owens and S. Owens, 2006. Examining the vascular pathway of sweet cherries grafted onto dwarfing rootstocks. HortScience 41: 674-679.

Olmstead, M. A.; N. Lang and G. Lang., 2010. Carbohy- drate profiles in the graft union of young sweet cherry trees grown on dwarfing and vigorous rootstocks. Scientia Horticulturae 124: 78-82.

Ojer, M.; G. Reginato, C. Ruitti, C. Arjona y O. Carrasco, 2015. Portainjertos y calidad de plantas. En: Tacchini, F. M., (Eds): Producción y comercialización de cereza en Mendoza, Argentina. Fundación Instituto de Desarrollo Rural. pp. 187-201.

Pedersen, B. H., 2005. Development of tensile strength in compatible and incompatible sweet cherry grafting. Canadian Journal of Botany 83: 202-210.

Pimentela, P.; R. D. Almadaa, A. Salvatierra, G. Toroa, M. Arismendia, M. Pinoa, B Sagredoa and M. Pintoa, 2014. Physiological and morphological responses of Prunus species with different degree of tolerance to long-term root hypoxia. Scientia Horticulturae 180: 14-23.

Pina, A. and P. Errea, 2005. A review of a new advances in mechanism of graft compatibility incompatibility. Scientia Horticulturae 106:1-11.

Pina A.; P. Errea, A. Wünsch and R. Gella, 2011. 'Monrepos', a plum rootstock for cherries. Hortscience 46 (2):322-323.

Prassinos, C.; J. Ko, G. Lang, A. lezzoni and K. Han, 2009. Rootstock induced dwarfing in cherries is caused by differential cessation of terminal meristem growth and is triggered by rootstock specific gene regulation. Tree Physiology 29: 927-936.

Rozpara, E.; Z. Grzyb and H. Zdyb, 1998. Growth and fruiting of two sweet cherry cultivars with different interstems. Acta Horticulturae 468: 345-352.

Sansavini S. and S. Lugli, 2014. New rootstocks for intensive sweet cherry plantations. Acta Horticulturae 1020: 317-320

Santos, A.; R. Santos-Ribeiro, J. Cavalheiro, V. Cordeiro and J. Lousada, 2006. Initial growth and fruiting of "Summit" sweet cherry (Prunus avium) on five rootstocks. New Zealand Journal of Crop and Horticultural Science 34: 269-277

Silva, H. y J. Rodriguez, 1995. Eficiencia y cálculo de la dosis de la fertilización nitrogenada. En: Bonomelli, C. (Ed.) Fertilización de plantaciones frutales. Chile, Facultad de Agronomía. Pontificia Universidad Católica de Chile. pp. 177-212.

Sitarek, M.; Z. Grzyb and B. Omiecinska, 2005. Performance of sweet cherry trees on Gisela 5 rootstock. Acta Horticulturae 667: 389-391.

Smith M. 2008. Relationship of trunk size to selected canopy size parameters for native pecan trees. Hortscience, 43(3):784-786.

Stehr, R., 2001. Experiences with dwarfing sweet cherry rootstocks in Northern Germany. Acta Horticulturae 667: 173-177. 
Usenik, V.; N. Fajt and F. Stampar, 2006. Effects of rootstocks and training system on growth, precocity and productivity of sweet cherry. Journal of Horticultural Science and Biotechnology 81(1): 153-157

Webster, A. and H. Schmidt, 1996. Rootstocks for sweet and sour cherries. En: Webster A. y N. Looney (Eds): Cherries: crop physiology, production and uses. CAB International. pp. 127-163.

Weibel, A. M., 2008. Dwarfing mechanisms of Prunus species as interstems and rootstocks on peach (Prunus persica $(\mathrm{L})$ Batsch) tree vegetative growth and physiology. Clemson University.

Wertheim, S.; J. Balkhoven, O. Callesen, J. Claverie, J. Vercammen and J. Ystaas, 1997. Results of two international cherry rootstock trials. Acta Horticulturae 468: 249-263.

Whiting, M.; G. Lang and D. Ophardt, 2005. Rootstocks and training system affect sweet cherry growth, yield and fruit quality. HortScience 40: 582-586.

Yano, T.; H. Inoue, Y. Shimizu and S. Shinkai, 2002. Dry matter partitioning and carbohydrate status of "Kawanakajima Hakuto" peach trees grafted onto different rootstocks or with an interstock at pre-bloom period. Journal of the Japanese Society for Horticultural Science 71 (2):164-170. 